\title{
A Continuous and Quantitative Analysis of the Uneven Distribution of Myocardial Blood Flow in Dogs Using a Distribution Function of the Glearance Time Constant
}

\author{
Kouichi Ashikawa, Eiji Ino-Oka, Shigenori Kitaoka, \\ Nobumasa Ishide, Kenji Tamaki, Shogen Isoyama, Taizo \\ Yanagiya, Takao Okubo and Tamotsu Takishima \\ The First Department of Internal Medicine, Tohoku University \\ School of Medicine, Sendai 980
}

\begin{abstract}
Ashikawa, K., Ino-OKa, E., Kitaoka, S., Ishide, N., Tamaki, K., Isoyama, S., Yanagiya, T., Okubo, T. and Takishima, T. A Continuous and Quantitative Analysis of the Uneven Distribution of Myocardial Blood Flow in Dogs Using a Distribution Function of the Clearance Time Constant. Tohoku J. exp. Med., 1982, 136 (2), 177-194-A continuous and quantitative analysis of the uneven distribution of coronary blood flow was accomplished in anesthetized open-chest dogs using the distribution function of the hydrogen $\left(\mathrm{H}_{2}\right)$ clearance time constant. The distribution function was derived by analysing the $\mathbf{H}_{\mathbf{2}}$ washout curves in the coronary sinus which were obtained by the $\mathbf{H}_{2}$ clearance method, using platinum electrodes placed in the coronary sinus. Twelve to seventeen platinum electrodes were employed to obtain simultaneous measurements of the regional myocardial flow before and after stenosis of the left anterior descending coronary artery. Theoretically, the $\mathrm{H}_{2}$ washout curves in the coronary sinus could be interpreted as a function with multi-exponential characteristcs, represented by an equation similar to the Laplace transform of the distribution function of the $\mathrm{H}_{2}$ clearance time constant. Therefore, we assumed that such an equation would represent the uneven distribution of coronary blood. We obtained the distribution function by using an approximation method to solve the integral equation and we employed digital computation to increase the resolution. Before stenosis of the left anterior descending coronary artery, the log distribution function curves with respect to the $\mathrm{H}_{2}$ clearance time constant were roughly symmetrical in terms of the maximum peak in the time constant. After stenosis of the left anterior descending coronary artery, the log distribution function curves had two peaks and the range of distribution was much larger than that before coronary stenosis. Our findings may prove to be valuable as a technique to estimate continuously and quantitatively the heterogeneous distribution of myocardial blood flow. myocardial blood flow; quantitative analysis; distribution function; hydrogen clearance time constant; coronary sinus
\end{abstract}

In the past decade, the heterogeneous distribution of the myocardial blood flow has been analyzed by a variety of methods; the use of radioactively labeled microspheres, the radioactive inert gas clearance technique, and a method which used noninvasive myocardial perfusion scintigraphy.

Received for publication, April 22, 1981. 
The use of radioactively labeled microspheres, a technique widely employed for this purpose, has several advantages over other methods, but it is limited in its applicability to experimental animals.

On the other hand, the radioactive inert gas clearance technique, using a detector which has a multiple colimeter, has been used for investigations with experimental animals and has even been applied to patients with coronary artery disease. However, there are limitations with respect to the continuous estimation of regional myocardial blood flow due to the compartmental approach inherent in the method.

The method which uses noninvasive myocardial perfusion scintigraphy, which has also been used for experimental animals and likewise applied to patients with coronary heart disease, is quite useful for qualitative estimation of the uneven distribution of myocardial perfusion, but it is not currently adequate for quantitative analysis.

The purpose of this study is to estimate continuously and quantitatively the uneven distribution of myocardial blood flow in anesthetized open-chest dogs. Therefore, we obtained coronary sinus hydrogen washout curves and analyzed them in terms of a continuous distribution function for estimating the myocardial volume. We did this by applying a procedure analogous to that which has been used for lung investigations and which has been previously reported by our laboratory (Nakamura et al. 1966; Okubo and Lenfant 1968).

The results of the distribution function were then compared with those obtained from both a conventional compartmental analysis and a mapping of regional myocardial blood flow.

\section{Methods}

\section{Experimental procedures}

Experiments were performed on six adult mongrel dogs, weighing between 13 and $19 \mathrm{~kg}$, which were anesthetized with sodium pentobarbital $(30 \mathrm{mg} / \mathrm{kg}$, i.v.). The animals were ventilated with room air via an endotracheal tube connected to an intermittent positive pressure respirator. A left lateral thoractomy was performed through the 4th intercostal space, and the heart was suspended in a pericardial cradle. A catheter was placed in the ascending aorta to measure aortic pressure. The heart was monitored by a standard limb lead electrocardiogram.

The left anterior descending coronary artery was isolated approximately $2 \mathrm{~cm}$ from its origin just below the first diagonal branch, by a silk thread which was loosely placed there. Heparin $(100 \mathrm{IU} / \mathrm{kg})$ was then administered intravenously. A solid plastic cannula was inserted into the ostium of the coronary sinus via the right atrial appendage. This cannula had a spindle-shaped swelling and a multi-holed tip to permit adequate flow and to avoid blood leakage. The inner diameter of the cannula was $4 \mathrm{~mm}$. The coronary venous return blood was directed to the superior vena cava, and the coronary sinus blood flow was measured by an electromagnetic flowmeter which was set in this circuit (Fig. 1).

The technique of measuring regional myocardial blood flow by the hydrogen gas clearance method has been described in previous reports from this laboratory (Inooka et al. 1970) and only the essential details will be described here. Twelve to seventeen platinum electrodes, $200 \mu \mathrm{m}$ in diameter with platinum-platinized tips $1 \mathrm{~mm}$ in length, were placed through the epicardium into the middle layer of the anterior wall of the left ventricle in three dogs (schematically shown in Fig. 3). Each electrode was placed about 10 to $15 \mathrm{~mm}$ apart. 


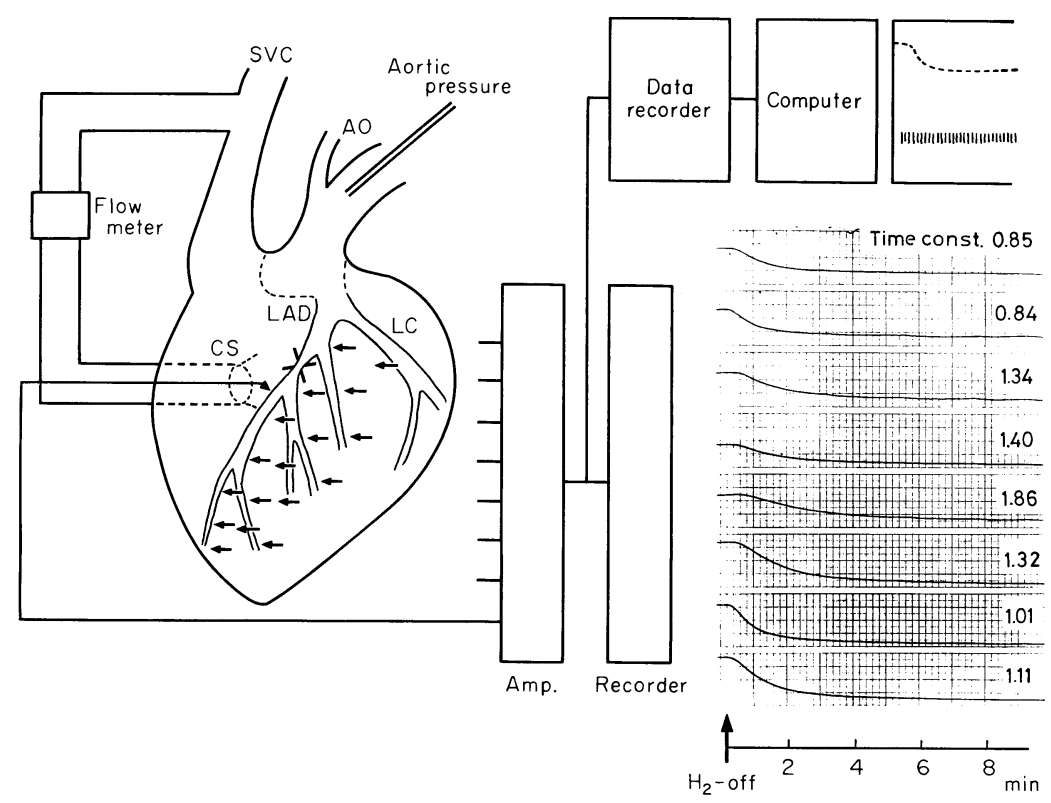

Fig. 1. A schematic diagram of the experimental preparation. AO, aorta; CS, coronary sinus; LAD, left anterior descending coronary artery; LC, left circumflex artery; SVC, superior vena cava.

Another platinum electrode was inserted into the coronary sinus via the coronary sinus cannula. These electrodes were connected to a high-gain, low-resistance differential amplifier. The silver reference electrode from the amplifier was attached to the animal subcutaneously in order to complete the physiological circuit. A positive potential of $0.4 \mathrm{~V}$ was applied to the reference electrode, as suggested by Aukland et al. (1964, 1967). The current produced in the circuit was recorded on a multichannel rectigraph (San-Ei Instruments, Tokyo).

The animals breathed hydrogen from the side channel of the endotracheal tube until myocardial tissue saturation was obtained. Saturation was recognized by the recording of stable plateaus of current produced at the site of the electrodes. The administration of hydrogen gas was then stopped. The desaturation currents were recorded and monitored until they returned to the baseline level (usually 6 to $8 \mathrm{~min}$ and 10 to $15 \mathrm{~min}$ before and after stenosis, respectively).

This procedure was carried out before and after stenosis of the coronary artery. The stenosis of the coronary artery was produced by a partial ligation of the left anterior descending coronary artery which was performed by overtying and then removing a 20gauge needle. The coronary sinus blood flow was maintained at a constant rate by means of intravenous drip-infusion of blood and/or saline. The regional myocardial blood flow was derived from the slope of the clearance curves obtained from each electrode. The recorded clearance curves were plotted on semilogarithmic paper with units of current deflection and time as the coordinates. Flow in $\mathrm{ml} / \mathrm{min} / 100 \mathrm{~g}$ of tissue was calculated according to the formula: Flow $=69.3 / T_{1 / 2}$ (Aukland et al. 1964), where $T_{1 / 2}$ is the half-time in $\min$ of the clearance curve. The tissue/blood partition coefficient and the specific gravity of the myocardium was assumed to be 1.00 .

The hydrogen washout curves from coronary sinus were recorded on the data recorder (Nihon Kohden RMG-5104, Tokyo), and reproduced on an $X-Y$ recorder (Model D-51, Riken Denshi Co., Ltd., Tokyo). We confirmed that there was no right atrial contamination of the coronary sinus blood due to the injection of hydrogen-saturated saline into the 
right atrium. Furthermore, the position of each electrode was checked at the end of each experiment. The heart was then excised. The ischemic area was identified by injecting Indocyanine Green via a polyethylene tube into the distal stenotic coronary artery, and the weight of the left ventricular free wall and the ischemic area were then measured.

Since hydrogen gas is highly diffusible in tissue, we can consider it to be both evenly distributed and instantly mixed when it is perfused in tissue water. Therefore, we can represent the even distribution of myocardial blood flow by the following formula:

$$
V \cdot C(t)=V_{H_{2}}(t)=V \cdot C(o) e^{-t / T}
$$

where

$C(t)=$ concentration of hydrogen after $t \mathrm{~min}$ in the myocardium

$C(o)=$ myocardial hydrogen concentration immediately before desaturation

$V=$ total myocardial volume

$V_{H_{2}}(t)=$ hydrogen quantity after $t$ min in the whole myocardium

$e=$ the base of the natural logarithm

$T=$ the clearance time constant which is the reciprocal of the amount of perfusion per min per unit of myocardial volume

Furthermore, the uneven distribution of myocardial blood can be idealized as so many tiny compartments of myocardial tissue are arranged in parallel, within which the perfusion of blood is even. Thus, we can consider each compartment to be perfused independently and homogeneously at its own perfusion rate; moreover, the total clearance rate of hydrogen at any given time in such a system would be the sum of these individual clearance rate.

Therefore, we can generalize equation (1) to represent the uneven distribution of myocardial blood flow as follows:

$$
V_{H_{\mathbf{2}}}(t)=\sum_{k=1}^{n} V k \cdot C(o) e^{-t / T k}
$$

where

$V k=$ myocardial volume of the $k$ th subdivision

$T k=$ time constant of the $k$ th subdivision, i.e. $V k / Q k$ where $Q k=$ the flow rate of the $k$ th subdivision

By considering the continuous distribution of the clearance time constant between zero and infinity, and giving the following domain to $T k$

$$
T k-\varepsilon<T k<T k+\varepsilon \quad(|\varepsilon| \ll \text { small })
$$

and defining

$$
V k=F(T k) \Delta T,
$$

equation (2) may be written as a limit in this form:

$$
\begin{aligned}
V_{H_{2}}(t) & =\lim _{n \rightarrow \infty} \sum_{k=1}^{n} V k \cdot C(o) e^{-t / T k} \\
& =\lim _{n \rightarrow \infty} \sum_{k=1}^{n} F(T k) \cdot C(o) e^{-t / T k} \Delta T \\
& =C(o) \int_{0}^{\infty} F(T) e^{-t / T} \mathrm{~d} T
\end{aligned}
$$

in which $F$ is defined by

$$
V_{H_{2}}(o)=\int_{0}^{\infty} F(T) \mathrm{d} T
$$


and in which $F(T)$ represents the distribution of the myocardial volume as a function of the time constant. And, with a lcgarithmic time scale, we can express the logarithmic distribution function $F^{\prime}(\log T)$ with a definition of $F(T) \mathrm{d} T=F^{\prime}(\log T) \mathrm{d} \log T$ as follows:

$$
V_{H_{\mathbf{2}}}(t)=C(o) \int_{-\infty}^{\infty} F^{\prime}(\log T) e^{-t / T} \mathrm{~d} \log T
$$

Since this expression can be rewritten in the form of a Laplace transform, we can calculate the distribution function $F^{\prime}(\log T)$ in equation (4) by using the approximation method for analysis of the inverted Laplace transform (Gross 1948; Schwarzl and Staverman 1956). The mathematical bases of these procedures have been previously reported from our laboratory (Nakamura et al. 1966). However, we have used digital computation here in an effort to increase the resolution of our approximation method. The advantages and disadvantages of digital resolution has been described in detail by Okubo and Lenfant (1968).

\section{Formulation of data}

When the myocardium is saturated with hydrogen and then perfused with hydrogenfree blood, the elimination of myocardial hydrogen can be described as follows:

$$
\begin{aligned}
V_{H_{\mathbf{2}}}(t) & =\int_{0}^{\infty} Q \cdot C s(t) \mathrm{d} t-\int_{0}^{t} Q \cdot C s(t) \mathrm{d} t \\
& =Q\left(\int_{0}^{\infty} C s(t) \mathrm{d} t-\int_{0}^{t} C s(t) \mathrm{d} t\right)
\end{aligned}
$$

where

$V_{H_{\mathbf{2}}}(t)=$ quantity of hydrogen after $t$ min in the whole myocardium

$Q=$ coronary sinus flow

$C s(t)=$ concentration of hydrogen after $t$ min in the coronary sinus

In practice, $\int_{0}^{\infty} C s(t) \mathrm{d} t$ was calculated as the area under hydrogen washout curve of the coronary sinus from $t=0$ to the end of the washout (usually 6 to $15 \mathrm{~min}$ );

moreover, $\int_{0}^{t} C s(t) \mathrm{d} t$ was obtained by sampling graphically the data every 10 sec. We then replotted the information thus derived.

Furthermore, in the equations $(2)$ and $(5), V_{H_{2}}(t)$ can also be written as

$$
\begin{aligned}
V_{H_{\mathbf{2}}}(t) & =Q\left(\int_{0}^{\infty} C s(t) \mathrm{d} t-\int_{0}^{t} C s(t) \mathrm{d} t\right) \\
& =\Sigma V k \cdot C(o) e^{-t / T k}
\end{aligned}
$$

where $Q \int_{0}^{\infty} C s(t) \mathrm{d} t$ represents the total volume of hydrogen in the system which has been washed out into the coronary sinus. Then, $\mathrm{d} V_{\mathrm{H}_{2}}(t) / \mathrm{d} t$ can be derived from equation (6) as follows :

$$
Q \cdot C s(t)=\Sigma Q k \cdot C(o) e^{-t / T k}
$$

Thus, the hydrogen washout cruve can be expressed as

$$
\frac{C s(t)}{C(o)}=\sum \frac{Q k}{Q} e^{-t / T k}
$$

As shown in Fig. 4 the hydrogen washout curve of the coronary sinus became multiexponential after stenosis of the coronary artery. However, these multi-exponential hydrogen washout curves can be resolved into mono-exponential components by the peeling 
process. Since the $T k$ and the $Q k / Q$ in equation (7) can be thus derived, the relative size of each component can be expressed as follows:

$$
\frac{V k}{V}=\frac{T k \cdot Q k}{\sum T k \cdot Q k}=\frac{T k \cdot Q k / Q}{\sum T k \cdot Q k / Q}
$$

\section{Rusults}

\section{Hemodynamic changes}

The general hemodynamic findings in 4 dogs are summarized in Table 1. After stenosis of the left anterior descending coronary artery, the aortic pressure and the coronary sinus flow increased slightly, while the heart rate showed a slight decrease. However, neither of these changes were statistically significant. We discarded the results of two in six experiments from the present study because of the extensive hemodynamic changes which took place after coronary stenosis; one due to hypotension and the other due to atrioventricular block.

TABLE 1. Hemodynamic data before and after stenosis of the left anterior descending coronary artery

\begin{tabular}{ccccccccc}
\hline Dog & $\begin{array}{c}\text { Weight of left Perfusion } \\
\text { ventricular } \\
\text { free wall }(\mathrm{g})\end{array}$ & $\begin{array}{c}\text { Mrea } \\
(\mathrm{g})\end{array}$ & $\begin{array}{c}\text { Mean arterial } \\
\text { pressure }(\mathrm{mmHg})\end{array}$ & \multicolumn{2}{c}{$\begin{array}{c}\text { Heart rate } \\
\text { (beats/min) }\end{array}$} & \multicolumn{2}{c}{$\begin{array}{c}\text { Coronary sinus } \\
\text { flow (ml/min) }\end{array}$} \\
\hline 1 & 63 & 32 & 90 & 91 & 162 & 148 & 58 & 60 \\
2 & 65 & 31 & 113 & 124 & 134 & 122 & 62 & 70 \\
3 & 65 & 30 & 70 & 80 & 118 & 112 & 58 & 60 \\
4 & 85 & 35 & 65 & 67 & 108 & 102 & 86 & 91 \\
\hline
\end{tabular}

Regional myocardial blood flow

A typical myocardial hydrogen clearance curve before stenosis of the coronary artery is shown in Fig. 2a. The net changes of current are plotted in arbitrary units on a logarithmic scale against time, and the resultant slopes and half-time are shown in Fig. 2b. The linear fall in electrode current on this scale indicates a monoexponential decay of myocardial hydrogen concentration. Essentially the same desaturation curves were obtained in almost all the experiments.

Fig. 3 shows the distribution of regional myocardial blood flow in the left ventricle before and after stenosis of the left anterior descending coronary artery. As was expected, the regional myocardial blood flow in the ischemic area was decreased. On the contrary, the regional myocardial blood flow adjacent to the ischemic area apparently increased as compared with its state prior to coronary stenosis. However, the regional myocardial blood flow in the non-ischemic area was maintained essentially unchanged throughout the experiment.

Compartmental analysis of the myocardium as a function of hydrogen clearance by the Coronary sinus hydrogen washout curve technique

Fig. 4 shows the net electrode current in the coronary sinus, normalized by the initial hydrogen current and plotted on a semilogarithmic scale against time 
a)

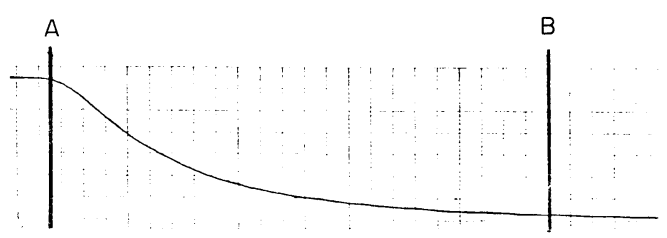

b)

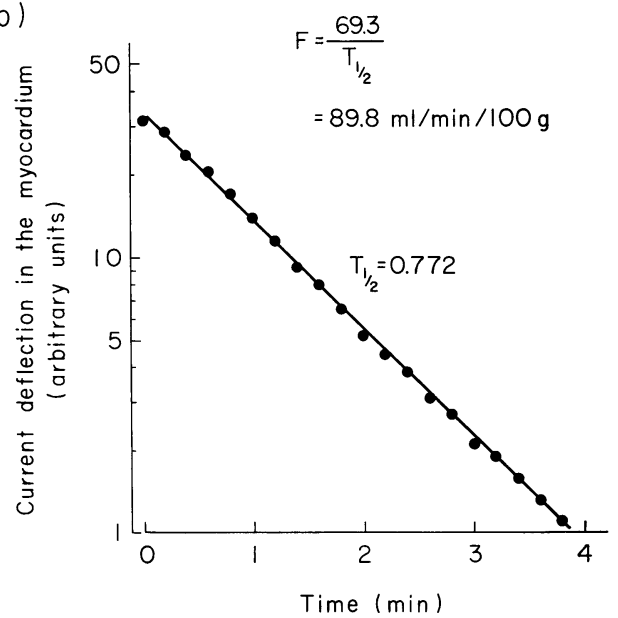

Fig. 2. a: A recording of the desaturation current obtained from the myocardium before stenosis of the left anterior descending coronary artery. A and B show the beginning and the completion of desaturation, respectively. b: A desaturation curve plotted on semilogarithmic axes.

before and after stenosis of the left anterior descending coronary artery in four experiments. The linear fall of electrode current on this scale represents a monoexponential decay of the coronary venous hydrogen concentration before coronary stenosis. However, after stenosis, the coronary sinus washout curves appeared in a different configuration. The deviation became apparent after the hydrogen concentration fell below 10 to $15 \%$ of its initial value. These deviated washout curves could be closely approximated by several exponential components which we designated as "fast", "middle" and "slow" phases of clearance as shown in Fig. 5. These findings suggest the presence of differently perfused components in the drainage area of the coronary sinus. The relative size of each perfusion area, calculated by using equation (7), is also shown in Fig. 5. This peeling process indicates that the coronary blood flow was distributed to three different perfusion areas in different amounts. The most highly perfused tissue area had a hydrogen clearance time constant of $0.8 \mathrm{~min}$ and represented $20 \%$ of the total area of perfusion. The second area of perfusion had a time constant of $1.2 \mathrm{~min}$ and represented $33 \%$ of the total area of perfusion. The most poorly perfused area had a time constant of $4.3 \mathrm{~min}$ and represented $47 \%$ of the total area of perfusion.

The equations for the hydrogen washout curves of the coronary sinus for each animal are presented in Table 2 as generalized forms of equation (6). Table 3 


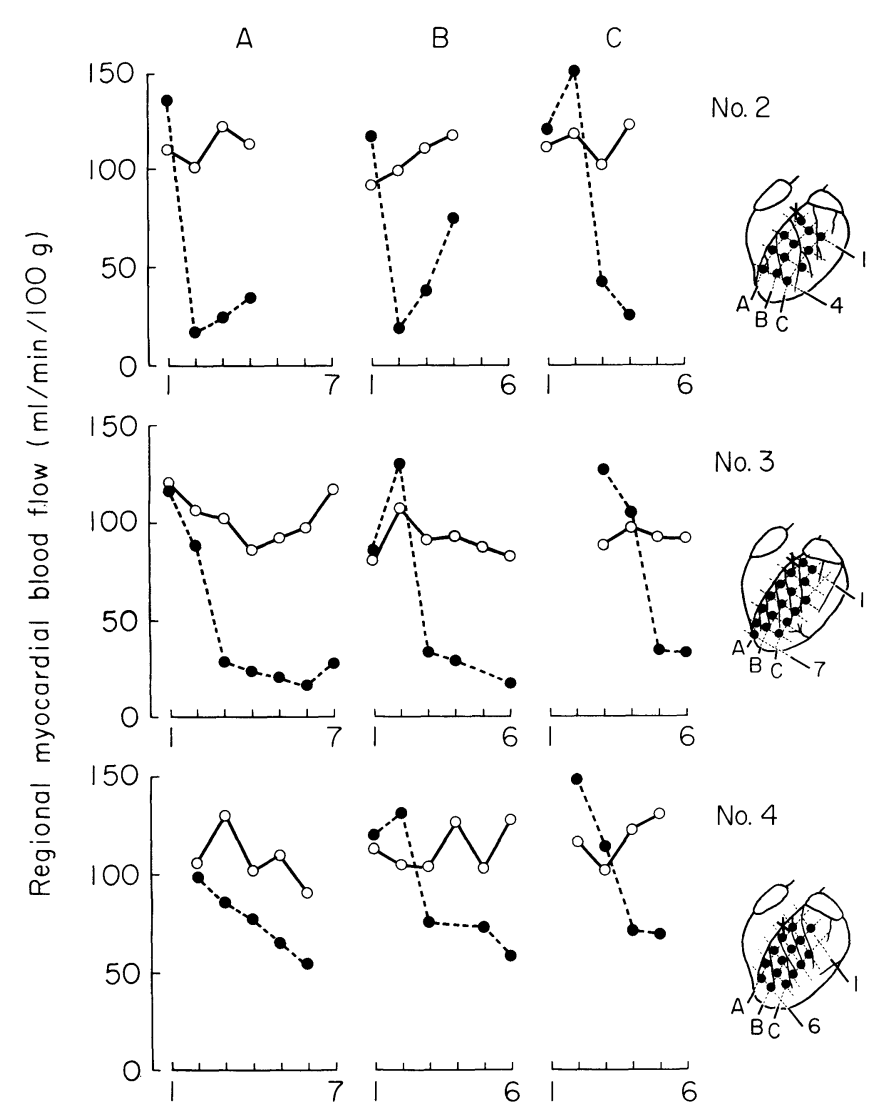

Fig. 3. Distribution of regional myocardial blood flow in the left ventricle. The insets show the arrangement of the measuring points: The electrodes are placed 10 to $15 \mathrm{~mm}$ apart and they form a grid of 3 rows (A, B, C) and 4 to 7 columns. The graphs show the distribution of the regional myocardial blood flow along the 3 rows before (open circles) and after (solid circles) stenosis of the left anterior descending coronary artery.

shows the clearance time constants and the relative size of the myocardium that is associated with them. Thus, although the conventional peeling process enables us to examine only one point at a time, we can speculate that the perfusion of the myocardium is a variable but a continuous process.

Distribution of myocardial volume as a function of hydrogen clearance time constant by means of mapping of the regional myocardial blood flow

The relative volume of each compartment of the myocardium at the site of each intramyocardial electrode was calculated in practice as follows:

$\begin{aligned} & \text { relative volume at } \\ & \text { each electrode }\end{aligned}=\frac{\text { weight of ischemic area }}{\begin{array}{l}\text { number of electrodes } \\ \text { in the ischemic area }\end{array}} /$ weight of the LV free wall 


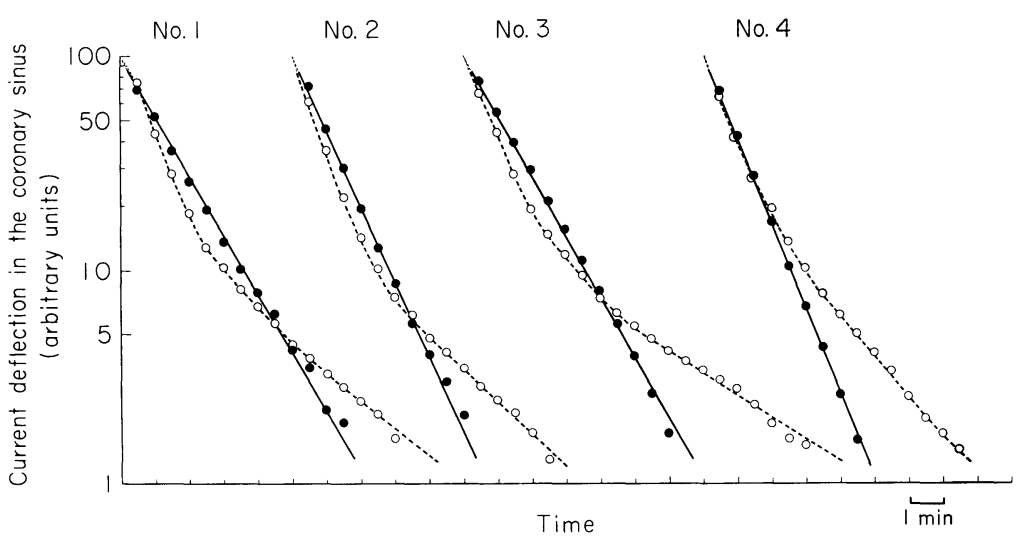

Fig. 4. Desaturation curves of the coronary sinus from the four experiments, normalized by the initial current, plotted on a semilogarithmic scale against time before (solid circles) and after (open circles) stenosis of the left anterior descending coronary artery. After coronary stenosis, the desaturaticn curves apparently deviated from the single exponential function form.

\begin{tabular}{ccc}
$\begin{array}{c}\text { Flow rate of } \\
\text { each component }\end{array}$ & $\begin{array}{c}\text { Clearance time } \\
\text { constant }(\mathrm{min})\end{array}$ & $\begin{array}{l}\text { Relative size of } \\
\text { each component }\end{array}$ \\
\hline$\frac{Q_{1}}{Q}=17$ & $T_{1}=4.30$ & $\frac{V_{1}}{V}=0.47$ \\
$\frac{Q_{2}}{Q}=43$ & $T_{2}=1.20$ & $\frac{V_{2}}{V}=0.33$ \\
$\frac{Q_{3}}{Q}=40$ & $T_{3}=0.80$ & $\frac{V_{3}}{V}=0.20$ \\
Total $=100$ & & Total $=1.00$ \\
\hline
\end{tabular}

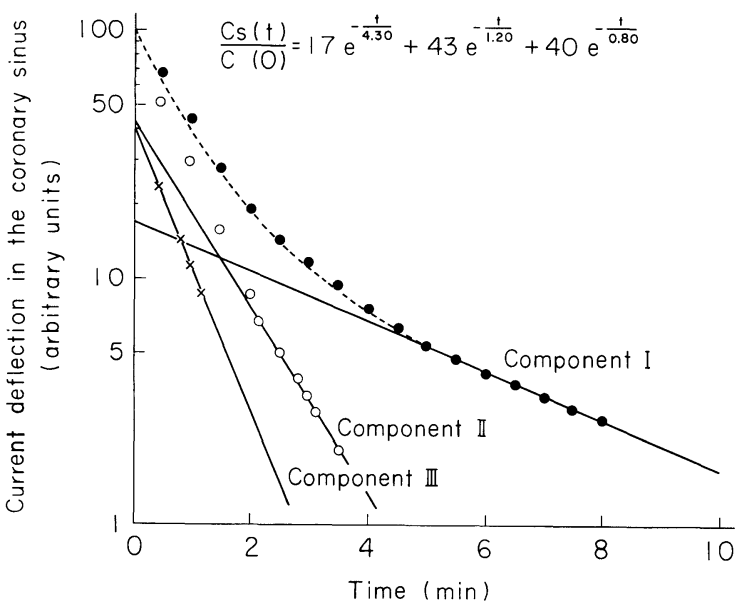

Fig. 5. A normalized desaturation curve of the coronary sinus composed of three monoexponential components, revealed by the peeling process (dog $\sharp 3)$. See text for details. 
TABLE 2. Net washout of hydrogen in the coronary sinus after stenosis of the left anterior descending coronary artery

$\begin{array}{cl}\text { Dog } & \frac{C s(t)}{C(o)}=\frac{Q \bar{k}}{Q} e^{-t / K T} \\ 1 & \frac{C s(t)}{C(o)}=18 e^{-t / 3.47}+43 e^{-t / 1.07}+39 e^{-t / 0.83} \\ 2 & \frac{C s(t)}{C(o)}=19 e^{-t / 2.93}+60 e^{-t / 0.80}+21 e^{-t / 0.63} \\ 3 & \begin{array}{l}C s(t) \\ C(o)\end{array}=17 e^{-t / 4.30}+43 e^{-/ 1.20}+40 e^{-t / 0.80} \\ 4 & \frac{C s(t)}{C(o)}=25 e^{-t / 2.60}+75 e^{-t / 0.87}\end{array}$

Abbreviations: $C s(t)=$ concentration of hydrogen after $t \mathrm{~min}$ in the coronary sinus; $C(o)=$ myocardial hydrogen concentration immediately before washout; $Q k=$ flow of $k$ th subdivision; $Q=$ total flow; $T k=$ time constant of $k$ th subdivision.

TABLE 3. Hydrogen clearance in the myocardium: the clearance time constant and the area of perfusion

\begin{tabular}{|c|c|c|c|}
\hline \multirow[b]{2}{*}{ Dog } & \multirow{2}{*}{$\begin{array}{l}\text { Before stenosis } \\
\text { Clearance time } \\
\text { constant (min) }\end{array}$} & \multicolumn{2}{|c|}{ After stenosis } \\
\hline & & $\begin{array}{l}\text { Clearance time } \\
\text { constant }(\mathrm{min})\end{array}$ & $\begin{array}{l}\text { Relative size of } \\
\text { each component }\end{array}$ \\
\hline 1 & $T=1.63$ & $\begin{array}{l}T_{1}=3.47 \\
T_{2}=1.07 \\
T_{3}=0.83\end{array}$ & $\begin{array}{l}V_{1} / V=0.44 \\
V_{2} / V=0.33 \\
V_{3} / V=0.23\end{array}$ \\
\hline 2 & $T=1.23$ & $\begin{array}{l}T_{1}=2.93 \\
T_{2}=0.80 \\
T_{\mathbf{3}}=0.63\end{array}$ & $\begin{array}{l}V_{1} / V=0.48 \\
V_{2} / V=0.41 \\
V_{3} / V=0.11\end{array}$ \\
\hline 3 & $T=1.50$ & $\begin{array}{l}T_{1}=4.30 \\
T_{2}=1.20 \\
T_{3}=0.80\end{array}$ & $\begin{array}{l}V_{1} / V=0.47 \\
V_{2} / V=0.33 \\
V_{3} / V=0.20\end{array}$ \\
\hline 4 & $T=1.13$ & $\begin{array}{l}T_{1}=2.60 \\
T_{2}=0.87\end{array}$ & $\begin{array}{l}V_{1} / V=0.50 \\
V_{2} / V=0.50\end{array}$ \\
\hline
\end{tabular}

The lateral and posterior walls of the left ventricle were included in the non-ischemic area. Fig. 6 shows a normalized distribution of myocardial volume as a function of the hydrogen clearance time constant, before and after stenosis of the left anterior descending coronary artery, in 3 animals in which the mapping of myocardial blood flow was performed.

Before stenosis, the distribution of the myocardial volume was within a narrow range in terms of the clearance time constant, peaking at about $1 \mathrm{~min}$; however, after stenosis, the range of distribution progressively widened as the disparity between the values of the time constants increased. 

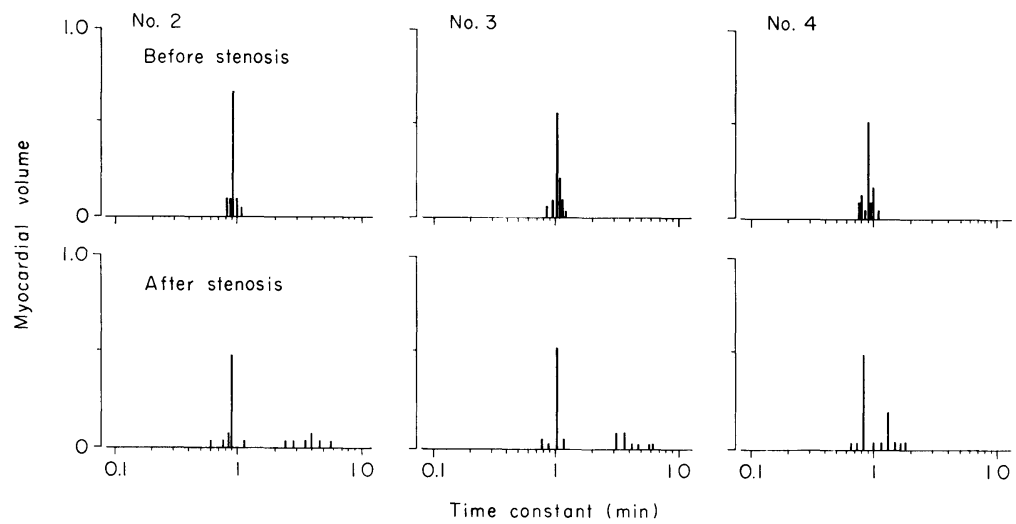

Fig. 6. The distribution of myocardial volume, nomalized by the total volume, as a function of the hydrogen clearance time constant before (upper row) and after (lower row) stenosis of the left anterior descending coronary artery. See text for details.

\section{Hydrogen clearance curves of the whole myocardium}

Fig. 7 illustrates the hydrogen clearance curves of the whole myocardium, replotted according to equation (5) and normalized by the initial values, as a function of time (log time), before and after stenosis of the left anterior descending coronary artery. After coronary stenosis the clearance curves showed a "fast" phase followed by a "slow" phase relative to their appearance before coronary stenosis.

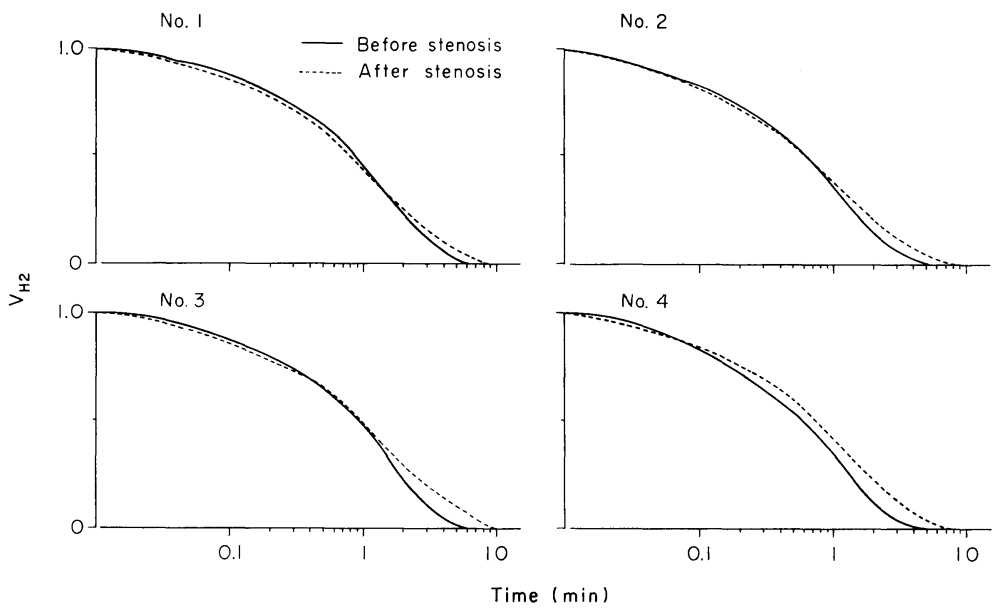

Fig. 7. Hydrogen clearance curves representing the time required for the total clearance within the whole myocardium in the drainage area of the coronary sinus, normalized by the initial values, as a function of time (log time) before and after stenosis of the left anterior descending coronary artery. After coronary stenosis, the clearance curves show a fast phase followed by a slow phase when compared to those before coronary stenosis. 


\section{Distribution function with respect to the hydrogen clearance time constant}

The distribution function curves obtained graphically by our approximation method for 4 animals are shown in Fig. 8. Before stenosis of the left anterior descending coronary artery, the curves had a maximum peak points at about $1 \mathrm{~min}$, and they were roughly symmetrical in shape, tapering off at about $6 \mathrm{~min}$. On the other hand, after stenosis, the curves appeared to be both flatter and wider, and they had a higher value of distribution at the high and low points of each time constant.

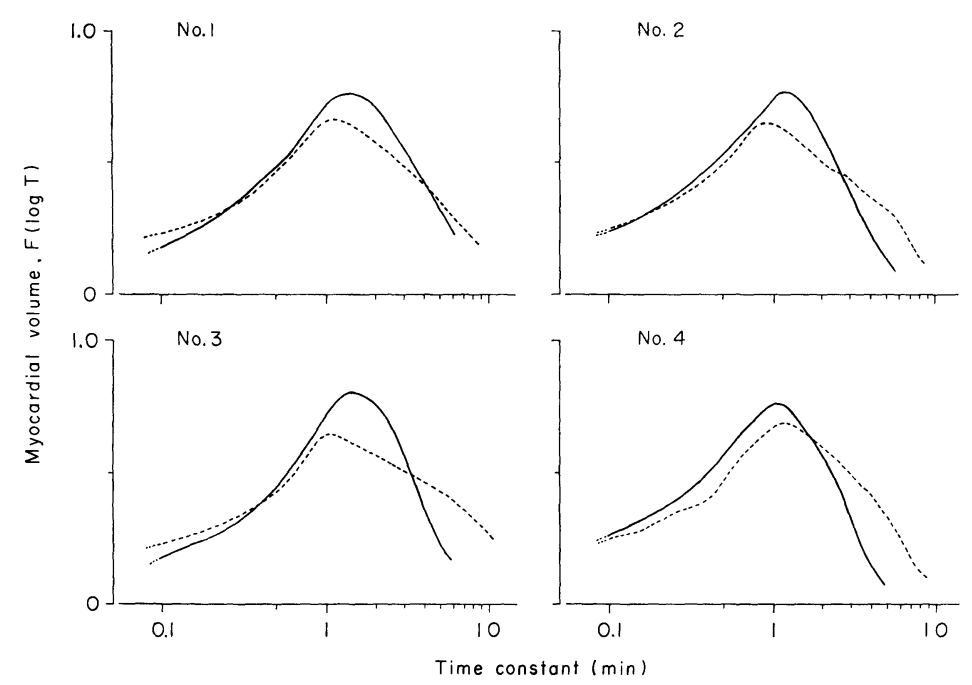

Fig. 8. Distribution function curves obtained graphically by an approximation method. Before coronary stenosis (solid line), the curves were roughly symmetrical with respect to clearance time constant of the maximum, about 1 min. After coronary stenosis (broken line), the curves are flattened and widened with an increase in distribution at the higher and lower values of the time constant.

Fig. 9 demonstrates the same pattern using digital computation to increase the order of resolution. Here, a "normalized" (i.e. the area under the curve equals one; thus, $\int_{0}^{\infty} F(T) \mathrm{d} T==1$ ) distribution of the myocardium is shown as a function of the time constant. All animals had a similar distribution function of myocardial volume before coronary stenosis. The curves were almost all symmetrical in the vicinity of the maximum peak, corresponding to the time constant value of about one min, and they had a relatively narrow base. This peak represents most of the myocardial volume. On the other hand, after stenosis, the distribution function was characterized by two distribution patterns: the first was characterized by the presence of two peaks; one in a time constant shorter than one min, and another in a time constant longer than one min. The second was characterized by a wide range of distribution due to a very slow return to the base line. 


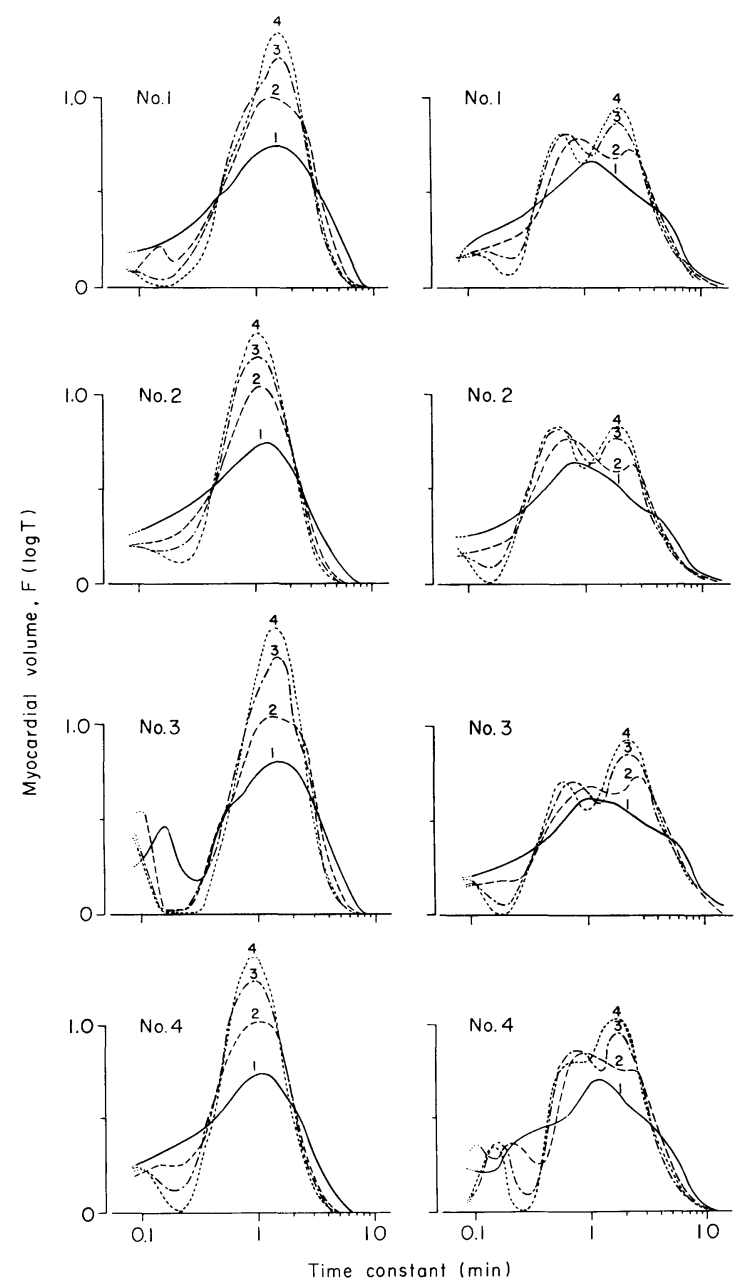

Fig. 9. The effects of increasing the order of approximation $(n=1-4)$ by digital computation on the shape of the myocardial volume distribution curves. The graphs on the left show the increased resolution power of the higher orders of approximation $(n=$ 3 or 4) before coronary stenosis. They have an almost symmetrical shape and a relatively narrow base. The graphs on the right show the changes in the distribution function curves after coronary stenosis; These curves are characterized by the presence of two peaks and a wider range of distribution, reflecting an increase in the volume of the myocardium which had slower clearance time constants.

\section{Discussion}

It is almost universally agreed that the average value of myocardial blood flow is difficult to evaluate when perfusion is non-uniformly distributed through the myocardium (Sullivan et al. 1967; Klocke et al. 1968; Klocke and Wittenberg 1969; Ganz et al. 1971). It is also reasonable that the concept of a continuous distribution of myocardial blood flow from the non-ischemic to the ischemic area as well as from 
the epicardium to the endocardium is more adequate and would provide more accurate results than an analysis of the myocardium based on the conceptualization of it as comprised of a limitted number of compartments, each of which has its own perfusion rate.

A number of methods have been used to estimate the distribution of myocardial blood flow in the intact and ischemic heart. The technique using Xenon-133 and a scintillation camera promised to be useful to estimate myocardial perfusion in multiple regions of the heart, because the washout of Xenon-133 from the myocardium could be directly interpreted as a function of tissue capillary blood flow (Cannon et al. 1975). This multicompartmental analysis, however, is expensive and discontinuous, and it does not yield quantitative results. Moreover, corrections must be made for the significant accumulation of Xenon-133 in the myocardial fat because of its high tissue-blood partition coefficient (Maseri et al. 1974). Furthermore, this technique generally does not provide images with high spatial resolution.

Recently, myocardial imaging methods using radioisotopic tracers have been described for the investigation of myocardial blood flow. Although the imaging methods are quite useful for a qualitative estimation of the uneven distribution of regional myocardial blood flow, they are inadequate to provide a continuous and quantitative estimation (Turner et al. 1976). The technique utilizing thallium-201 and ${ }^{99 m}$ Tc-PYP, which has recently become available for clinical use, does not always show good correlation between the intensity of scintigraphic abnormality and the distribution of regional myocardial blood flow (Marcus et al. 1967; Gewirtz et al. 1978). It has been suggested that biological variability plays an important role in the lack of sensitivity of the scintigrams (Mueller et al. 1976; Buja et al. 1977; Selwyn et al. 1978).

Furthermore, the intensity of the abnormalities which are shown on the cardiac scintigram in the ischemic heart may be affected by the complex three dimensional configuration of ischemia and the back ground radiation from surrounding tissue. Moreover, changes of the heart position associated with breathing may significantly blur the image by altering the spatial relationship between the heart and the detector. In addition, changes of the geometric relationship between the location of ischemia and the detector make quantitative evaluation nearly impossible. Another problem is that these scintigrams are usually taken in multiple projections at different distances from the heart. Thus, the intensity or "visibility" of the abnormality on the cardiac scintigram cannot serve as a sensitive and quantitative index of the myocardial perfusion rate because of the many variables described above.

Since none of the previously described methods proved to be sufficient for a continuous and quantitative estimation of the regional myocardial blood flow, we introduced a distribution function, which has been previously reported from this laboratory in connection with lung experiments (Nakamura et al. 1966), for this purpose. We obtained this distribution function by analyzing the hydrogen washout curves of the coronary sinus. 
The choice of hydrogen as a test gas was based both on its extremely low solubility in blood (i.e. the blood/gas partition coefficient $=0.015$ ) and on its high intrinsic diffusibility (Klocke et al. 1968). Because of its low solubility, elimination of the recirculated hydrogen from the mixed venous blood in the lung is more complete than it would be for other soluble gases (Chidsey et al. 1959). Furthermore, because of its low tissue-blood partition coefficient in fat, the concentration of hydrogen in the venous blood draining adipose tissue would also be very low compared with that of the venous blood draining tissue in the rest of the heart. Special care was taken, in the present study, to maintain sufficient ventilation to the lungs to assure that the ventilation-perfusion ratio was reasonably constant and within the normal range.

The circuit for recording the hydrogen desaturation curves was essentially the same as that described by Aukland et al. (1964), but we modified it slightly in order to obtain more stable recordings. Since the hydrogen current in the preliminary experiment was rather constant in the potential range of 0 to 0.4 volts, a positive potential of 0.4 volts was applied to the reference electrode to minimize the oxygen reduction at the platinum electrode. The mean time constant of this recording circuit was 12 sec. We confirmed that there was no contamination of venous blood in the coronary sinus of each heart by injecting hydrogen-saturated saline into the right atrium.

Before coronary stenosis, the hydrogen washout curves in the coronary sinus were reasonably close to a single exponential form, which suggested uniform coronary blood perfusion in the heart. On the other hand, the curves showed distinct deviations from a single exponential form after stenosis of the anterior descending coronary artery.

Klocke et al. (1968), using coronary venous hydrogen desaturation curves in a study with anesthetized dogs, have also demonstrated that semilogarithmically plotted desaturation curves did not form single exponentials after coronary occlusion. They also reasoned that this indicated heterogeneous myocardial perfusion, even though the curves came closest to forming single exponentials before the coronary occlusion occurred.

By the conventional graphical analysis, several components of the exponential washout curve may be defined; but then the heart looks as if it consists of two or three parallel compartments, each with its own perfusion rate, because of the limitation of this analytical technique. On the other hand, mapping the regional myocardial blood flow of the heart might be described as a multicompartmental approach. This mapping method would correlate more closely with the real distribution of regional myocardial blood flow. But, it is still not adequate for a continuous and quantitative analysis.

In this paper we have demonstrated that the hydrogen washout curves of the coronary sinus can be interpreted as a curve with a multiple exponential character as defined by the distribution function of the clearance time constant. Before coronary stenosis, the distribution of the myocardial volume with respect to the hydrogen clearance time constant was roughly symmetrical and it had a relatively 
narrow base. After stenosis of the left anterior descending coronary artery, the distribution curves of the myocardium, as a function of the hydrogen clearance time constant, showed two basic patterns: The first pattern was characterized by the presence of two peaks-one represented a short time constant corresponding to normal and high perfusion of the myocardium; the other represented a longer time constant corresponding to low perfusion of the myocardium. The second pattern was characterized by a wide range of distribution because of the slow return to the base line corresponding to an increase in the volume of the low-perfused myocardium. The high perfusion area adjacent to the acutely ischemic myocardium, which was demonstrated by mapping the regional myocardial blood flow (Fig. 3), has also been reported by other investigators (Rees and Redding 1969; Becker et al. 1973).

Since the area under the distribution function curve represents the total myocardial volume, the relative volume with respect to the time constant can be obtained. The final step of the mathematical analysis consists in inverting an equation resembling the Laplace transform with the aid of a certain approximation method. We obtained a higher order of resolution in the approximation method by using digital computation. Since the distribution function used in this study was a single-exponential equation before coronary stenosis, a perfect approximation should appear like a spike (as opposed to a peaked curve). Although, we were unable to produce the spiked appearance we had hoped for, we none the less found this computer-assisted approximation technique to be useful for continuous and quantitative analysis of the uneven distribution of myocardial blood flow. The advantages and disadvantages of our computer-assisted approximation technique have been extensively described elsewhere (Okubo and Lenfant 1968).

In conclusion, our study revealed the great advantage of continuous and quantitative analysis of the distribution of myocardial blood flow, particularly when it is applied to a heart where the distribution is uneven. In normal dog hearts, the typical shape of the distribution function curves of the myocardium with respect to the hydrogen clearance time constant was symmetrical in terms of a maximum peak with a narrow base. In ischemic hearts, there was a marked change of the distribution function curves, characterized by the presence of two peaks and by a wide range of distribution toward the low time constant.

The findings in this study may provide a technique which will be quite useful for continuously and quantitatively analyzing the uneven distribution of myocardial blood flow with respect to angiographic changes.

Only limitation of our analysis will be the drainage area for the coronary sinus. It has been reported that almost ninety-five percent of the coronary sinus blood comes from the perfusion area of the left coronary artery in dog (Hammond and Austen 1967; Tamura et al. 1969). In the study of postmortem human heart, Hood (1968) demonstrated that ninety-six percent of the venous drainage of the left ventricular free wall and the interventricular septum have a connection with the coronary sinus. 
Therefore, it is not reasonable to say that our analysis will have some definite but negligible technical limitation for detection of uneven distribution of blood flow within the left ventricle.

\section{References}

1) Aukland, K., Bower, B.F. \& Berliner, R.W. (1964) Measurement of local blood flow with hydrogen gas. Circulat. Res., 14, 164-187.

2) Aukland, K., Kiil, F., Kjekshus, J. \& Semb, G. (1967) Local myocardial blood flow measured by hydrogen polarography; Distribution and effect of hypoxia. Acta physiol. scand., 70, 99-111.

3) Becker, L.C., Ferreira, R. \& Thomas, M. (1973) Mapping of left ventricular blood flow with radioactive microspheres in experimental coronary artery occlusion. Cardiovasc. Res., 7, 391-400.

4) Buja, J.M., Tofe, A.J., Kulkarni, P.V., Mukherjee, A., Parkey, R.W., Francis, M.D., Bonte, F.J. \& Willerson, J.T. (1977) Sites and mechanisms of localization of technitum $-99 \mathrm{~m}$ phosphorus radiopharmaceuticals in acute myocardial infarcts and other tissues. J. clin. Invest., 60, 724-740.

5) Cannon, P.J., Sciacca, R.R., Fowler, D.L., Weiss, M.B., Schmidt, D.H. \& Casarella, W.J. (1975) Measurement of regional myocardial blood flow in man: Discription and critique of the method using Xenon-133 and a scintillation camera. Amer. J. Cardiol., 36, 783792.

6) Chidsey, C.A., III, Fritts, H.W., Jr., Hardewig, A., Richards, D.W. \& Cournand, A. (1959) Fate of radioactive krypton $\left(\mathrm{Kr}^{85}\right)$ introduced intravenously in man. J. appl. Physiol., 14, 63-66.

7) Ganz, W., Tamura, K., Marcus, H.S., Donoso, R., Yoshida, S. \& Swan, H.J.C. (1971) Measurement of coronary sinus blood flow by contiunous thermo dilution in man. Circulation, 44, 181-195.

8) Gewirtz, H., O'keefe, D.D., Pohost, G.M., Strauss, H.W., McIlduff, J.B. \& Daggeti, W.M. (1978) The effect of ischemia on thallium-201 clearance from the myocardium. Circulation, 58, 215-219.

9) Gross, B. (1948) On creep and relaxation. J. appl. Physiol., 19, 257-264.

10) Hammond. G.L. \& Austen, W.G. (1967) Drainage patterns of coronary arterial flow as determined from the isolated heart. Amer. J. Physiol., 212, 1435-1440.

11) Hood, W.B. (1968) Regional venous drainage of the human heart. Brit. Heart J., 30, $105-109$.

12) Inooka, E., Kanazawa, T., Suzuki, N., Takahashi, T., Kozuka, Y., Mori, A., Miura, M., Komatsu, Y., Maruyama, Y. \& Nakamura, T. (1970) Measurement of regional myocardial blood flow by hydrogen gas clearance method (Report 2); Its application to un-anesthetized dogs. Jap. Circulat. J., 34, 936. (abstr.)

13) Klocke, F.J. \& Wittenberg, S.M. (1969) Heterogeneity of coronary blood flow in human coronary artery disease and experimental myocardial infarction. Amer. J. Cardiol., 24, 782-790.

14) Klocke, F.J., Koberstein, R.C., Pittman, D.E., Bunnell, I.L., Greene, D.G. \& Rosing, D.R. (1968) Effects of heterogeneous myocardial perfusion on coronary venous $\mathrm{H}_{2}$ desaturation curves and calculations of coronary flow. J. clin. Invest., 47, $2711-2724$.

15) Marcus, M.L., Tomanek, R.J., Ehrhardt, L.C., Kerber, R.E., Brown, D.D. \& Abboud, F.M. (1967) Relationships between myocardial perfusion, myocardial necrosis, and tecnetium $-99 \mathrm{~m}$ pyrophosphate uptake in dogs subjected to sudden coronary occlusion. Circulation, 54, 647-653.

16) Maseri, A., Pesola, A., L'Abbate, A., Contini, C., Michelassi, C. \& D’Angelo, T. (1974) Contribution of recirculation and fat diffusion to myocardial washout curves obtained by external counting in man. Stochastic varsus monoexponential analysis. Circulat. Res., 35, 826-834. 
17) Mueller, T.M., Marcus, M.L., Ehrhardt, J.C., Chaudhuri, T. \& Abboud, F.M. (1976) Limitations of thallium-201 myocardial perfusion scintigrams. Circulation, 54, 640-646.

18) Nakamura, T., Takishima, T., Okubo, T., Sasaki, T. \& Takahashi, H. (1966) Distribution function of the clearance time constant in lungs. J. appl. Physiol., 21, 227-232.

19) Okubo, T. \& Lenfant, C. (1968) Distribution function of lung volume and ventilation determined by lung $\mathrm{N}_{2}$ washout. J. appl. Physiol., 24, 658-667.

20) Rees, J.R. \& Redding, V.J. (1969) Experimental myocardial infarction in the dog. Comparison of myocardial blood flow within, near, and distant from the infarct. Circulat. Res.. 24, 161-170.

21) Selwyn, A.P., Welmal, E., Pratt, T.A., Clark, J., McArthur, C. \& Lavender, J.P. (1978) The interpretation of thallium-201 cardiac scintigrams: Studies in experimental ischemic heart disease in dogs. Circulat. Res., 43, 287-293.

22) Schwarzl, F. \& Staverman, A.J. (1956) Higher approximation methods for the relaxation spectrum from static and dynamic measurements of viscoelastic materials. Appl. Sci. Res., Sect. A, 4, 127-141.

23) Sullivan, J.M., Taylor, W.J., Elliott, W.C. \& Gorlin, R. (1967) Regional myocardial blood flow. J. clin. Invest., 46, 1402-1412.

24) Tamura, K., Laks, M.M., Garner, D. \& Swan, H.J.C. (1969) Distribution rates of coronary inflow and outflow in the post-mortem canine heart. Cardiovasc. Res., 3, $324-330$.

25) Turner, J.H., Selwyn, A.P., Jones, T., Evans, T.R., Raphael, M.J. \& Lavender, J.P. (1976) Continuous imaging of regional myocardial blood flow in dogs using Krypton$81 \mathrm{~m}$. Cardiovasc. Res., 10, 398-404. 